\title{
Anaemia following Artemisinin-Based Combination Treatments of Uncomplicated Plasmodium falciparum Malaria in Children: Temporal Patterns of Haematocrit and the Use of Uncomplicated Hyperparasitaemia as a Model for Evaluating Late-Appearing Anaemia
}

\author{
Akin Sowunmi $^{\mathrm{a}-\mathrm{c}}$ Kazeem Akano $^{\mathrm{b}}$ Godwin Ntadom $^{\mathrm{a}}$ Adejumoke Ayede $^{\mathrm{d}}$ \\ Stephen Oguche ${ }^{a}$ e Chimere Agomo ${ }^{a, f}$ Henrietta Okafor ${ }^{a, g}$ Ismaila Watila ${ }^{a, h}$ \\ Martin Meremikwu ${ }^{a, i}$ William Ogala ${ }^{a, j}$ Philip Agomo ${ }^{a, f} \quad$ Elsie Adowoye ${ }^{k}$ \\ Bayo Fatunmbi $^{\mathrm{n}}$ Temitope Aderoyejel Christian Happi ${ }^{\mathrm{a}, \mathrm{m}}$ Grace Gbotoshob, c \\ Onikepe Folarin ${ }^{m}$ \\ ${ }^{a}$ Antimalarial Therapeutic Efficacy Monitoring Group, National Malaria Elimination Programme, The Federal \\ Ministry of Health, Abuja, bepartment of Pharmacology and Therapeutics, University of Ibadan, Ibadan, ${ }^{\mathrm{C}}$ Institute \\ of Medical Research, College of Medicine, University of Ibaden, Ibadan, ${ }^{d}$ Department of Paediatrics, University of \\ Ibadan, Ibadan, e Department of Paediatrics, University of Jos, Jos, ${ }^{\mathrm{f}}$ Nigeria Institute of Medical Research, Lagos, \\ ${ }^{9}$ Department of Paediatrics, Institute of Child Health, University of Nigeria Teaching Hospital, Enugu, h Department \\ of Paediatrics, Specialist Hospital, Maiduguri, 'Department of Paediatrics, University of Calabar, Calabar, \\ jDepartment of Paediatrics, Ahmadu Bello University, Zaria, ${ }^{\mathrm{k}}$ Department of Physiology, University of Ibadan, \\ Ibadan, 'Department of Clinical Pharmacology, University College Hospital, Ibadan, and 'm Department of Biological

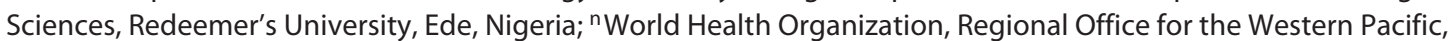 \\ Khan Daun Penh, Phnom Penh, Cambodia
}

\section{Keywords}

Anaemia - Uncomplicated hyperparasitaemia · Children ·

Artemisinin-based combination treatments · Nigeria

\section{Abstract}

Background: In severe malaria, intravenous artesunate may cause delayed haemolytic anaemia but there has been little evaluation of the propensity of oral artemisinin-based combination treatments (ACTs) to cause late-appearing anae-

\section{KARGER}

(C) 2017 S. Karger AG, Basel

E-Mail karger@karger.com

www.karger.com/che mia. Methods: The frequency of anaemia (haematocrit $<30 \%$ ), and temporal changes in haematocrit were evaluated in 1,191 malarious children following ACTs. "Haematocrit conservation" was evaluated by using the fall in haematocrit/1,000 asexual parasites cleared from the peripheral blood ( $\mathrm{FIH} / 1,000$ asexual parasites $\mathrm{cpb}$ ), and the ratio of the average haematocrit (on the first 3 days of starting treatment):total parasitaemia cleared. Results: The frequency of anaemia decreased significantly following treatment. FIH/1,000 asexual parasites cpb, average haema- 
tocrit:total parasitaemia cleared, and mean haematocrit 5 weeks after treatment began were significantly lower in hyperparasitaemic children than in children without hyperparasitaemia, suggesting haematocrit conservation during treatment followed later by a loss of haematocrit. Asymptomatic late-appearing anaemia occurred in $6 \%$ of the children. Conclusion: Artesunate-amodiaquine and artemether-lumefantrine contribute to haematocrit conservation at high parasitaemias but may cause late-appearing anaemia.

(c) 2017 S. Karger AG, Basel

\section{Introduction}

Artemisinin-based combination treatments (ACTs) have been adopted by over 100 countries for the treatment of uncomplicated Plasmodium falciparum malaria, and intravenous artesunate for severe malaria $[1,2]$. Intravenous artesunate treatment of severe malaria may cause delayed haemolytic anaemia $[3,4]$, which has been attributed to the destruction of once-infected (pitted) erythrocytes 7-21 days after pitting [3]. In patients with heavy parasitaemias or severe malaria, this process prevents a precipitous decline in haematocrit in the first few days following treatment. This is a life-saving process [3].

In endemic countries with poor resources where once-infected erythrocytes cannot be readily quantified, the extent of the fall in haematocrit following ACTs can be quantified by estimating the fall in haematocrit/1,000 asexual parasites cleared from the peripheral blood (FIH/1,000 asexual cpb) on the first 2 days of treatment [5]. Using this method, we showed that there is little or no fall in haematocrit following ACTs, particularly when parasitaemias are heavy, and this has been termed "haematocrit conservation" [5].

Uncomplicated $P$. falciparum hyperparasitaemia is common and responds therapeutically to ACTs $[6,7]$, but there has been little evaluation of its relationship with delayed or late-appearing anaemia and the timecourse of the frequency of anaemia in African children after oral ACTs.

This study evaluated the frequency of anaemia (haematocrit $<30 \%$ ) before and after ACTs and also the temporal changes in haematocrit in children with uncomplicated hyperparasitaemia and non-hyperparasitaemia following ACTs.

\section{Patients and Methods}

The initial study was conducted from October 2009 to November 2010 in malarious children under the age of 5 years in Nigeria: in Agbani, Barkin Ladi, and Damboa, in the states of Enugu, Plateau, and Borno, respectively (the eastern flank of the study sites), and also in Ijede, Ibadan, and Makarfi in the states of Lagos, Oyo, and Kaduna Nigeria, respectively (the western flank). The later study was conducted from 2009 to 2014 in malarious children $\leq 10$ years old in Ibadan. The details of the efficacy and gametocyte carriage study have been reported elsewhere $[8,9]$. The study profile is shown in Figure 1.

In the initial study, we assessed the under-5-year-olds with acute, symptomatic uncomplicated $P$. falciparum malaria and parasitaemias of 1,000-200,000 asexual forms/ $\mu \mathrm{L}$. In the later study, we assessed $\leq 10$-year-olds with parasitaemias of between 1,000 and $>250,000$ asexual forms/ $\mu \mathrm{L}$ (uncomplicated hyperparasitaemia). Patients with complicated hyperparasitaemia or other features of severe malaria, and those with severe malnutrition or sickle-cell anaemia were excluded from the study. The day of presentation was regarded as day zero. Patients were treated with artemether-lumefantrine $(\mathrm{AL})$ or artesunate-amodiaquine (AA) co-formulated for 3 days (days $0-2$ ) as previously described [9]. In the initial study, follow-up was done daily on days $1-3$ and on days $7,14,21$, and 28 , and this was extended to days 35 and 42 in the later study. The study protocol was approved by the National Health Research Ethics Committee, Abuja, and the Ministry of Health, Ibadan, Nigeria.

Parasitaemia was quantified, and fever clearance and parasite clearance times were determined as previously described $[9,10]$. Hyperparasitaemia was defined as asexual parasitaemia $>250,000 /$ $\mu \mathrm{L}$. Haematocrit was estimated before and after treatment and in all children seen at each follow-up day, and was referred to as "haematocrit level per visit day." Anaemia was defined as a haematocrit $<30 \%$, and was classified as mild, moderate, or severe at $21-29,15-$ 20 , or $<15 \%$, respectively. Early-appearing anaemia, late-appearing anaemia, and $\mathrm{FIH} / 1,000$ asexual parasites $\mathrm{cpb}$ following treatment were defined as previously described $[5,11,12]$. On days $0-2$, the ratio of average haematocrit:total parasitaemia cleared during the same period was also used as a broader index of "haematocrit conservation." Anaemia recovery time was defined as the time elapsing from the start of anaemia until haematocrit $\geq 30 \%$. The profile of investigations is shown in Figure 1. In hyperparasitaemic and nonhyperparasitaemic children matched for age, gender, treatment, and same-day presentation, temporal changes in haematocrit post-treatment initiation were classed as previously described [11]. A diagnosis of late-appearing anaemia was made as previously described [12] .

\section{Data Analysis}

Data was analysed using Epi Info ${ }^{\mathrm{TM}}$ software v7 [13] and the statistical program SPSS for Windows v20.0 [14]. Proportions were compared by calculating $\chi^{2}$ values using the Yates correction, the Fisher exact test, or the Mantel-Haenszel test. Normally distributed, continuous data was compared by means of the Student $t$ test and analysis of variance (ANOVA). For the analysis of haematocrit levels per visit day, post hoc comparisons were done using the Tukey honestly significant difference (Tukey HSD). Data not conforming to a normal distribution was compared by the Mann-Whitney $\mathrm{U}$ test and the Kruskal-Wallis test (or by Wilcoxon ranked sum test). $p$ values $<0.05$ were taken to indicate significant differences.
Sowunmi et al. 


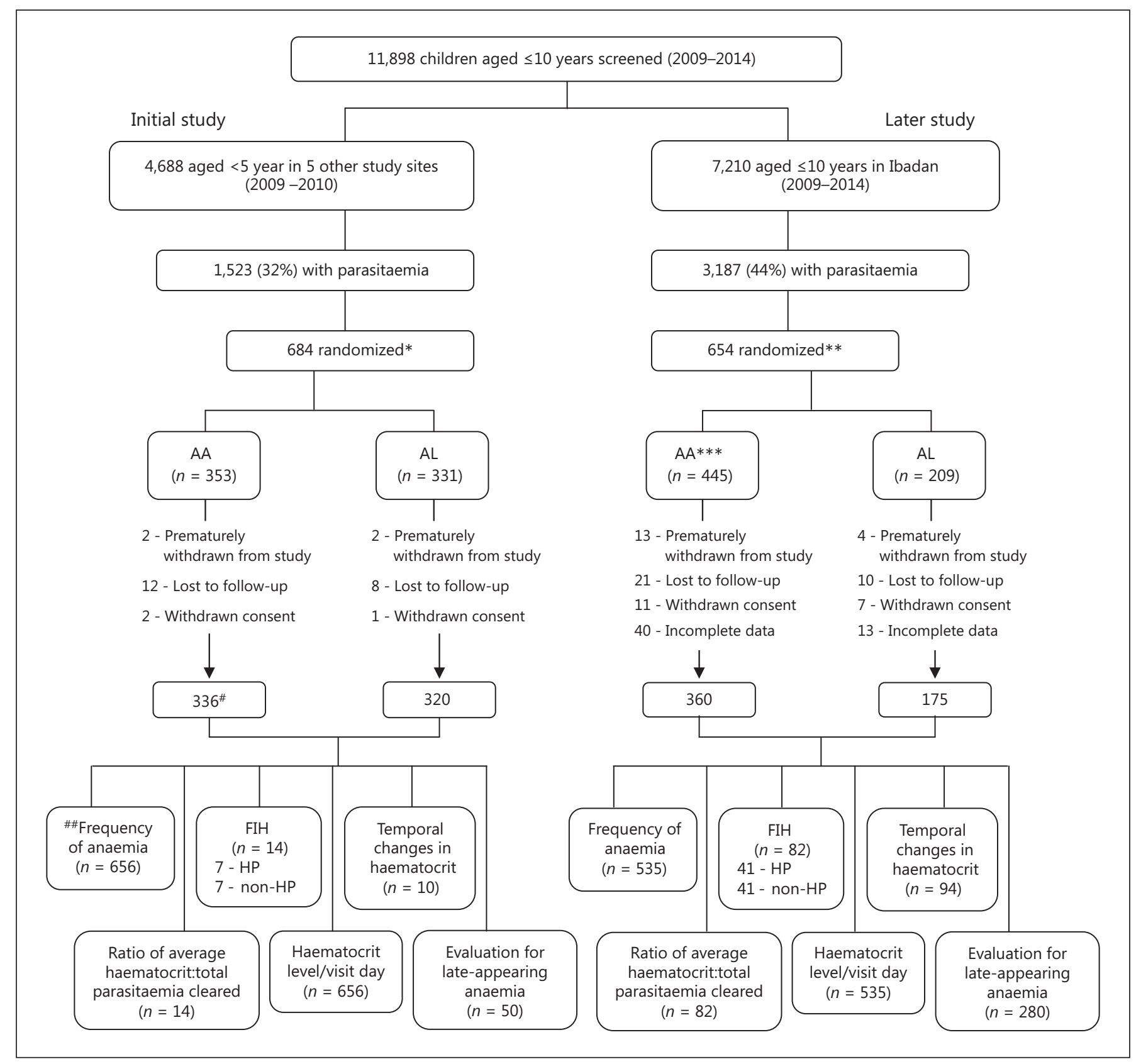

Fig. 1. Study profile and investigations carried out. AA, artesunate-amodiaquine; AL, artemether-lumefantrine; HP, hyperparasitaemic. * Follow-up was for 28 days, ${ }^{* *}$ follow-up was for 42 days, ${ }^{* *}$ randomization at a ratio of 2:1 for AA and AL, " number of children that completed follow-up period, \#\# investigations of children $<5$ years of age.

\section{Results}

The characteristics of the 1,191 children enrolled in the study are summarized in Table 1. At presentation, 423 children $(35.5 \%)$ were anaemic. Anaemia was mild, moderate, and severe in 380,41 , and 2 children, respectively.
In the initial study, at presentation, $70 / 109(65 \%)$, 67/113 (59\%), 27/110 (24\%), 43/119(36\%), 43/106 (41\%), and 43/99 (43\%) children in Damboa, Agbani, Barkin Ladi, Makarfi, and the Sabo quarters of Ibadan and Ijede, respectively, were anaemic. Anaemia was more frequently found in children from sites located on the eastern 
Table 1. Baseline characteristics of the children enrolled in the study

\begin{tabular}{|c|c|c|c|c|}
\hline Parameters & $\mathrm{AA}(n=696)$ & $\mathrm{AL}(n=495)$ & $\operatorname{ALL}(n=1191)$ & $p$ value \\
\hline Male/female, $n$ & $370 / 326$ & $272 / 223$ & $642 / 549$ & 0.58 \\
\hline Mean age, years & $4.8(4.6-5)$ & $4.2(4-4.4)$ & $4.5(4.4-4.7)$ & \\
\hline Age $<5$ years, $n$ & $428(61 \%)$ & $377(76 \%)$ & $813(68 \%)$ & $<0.0001$ \\
\hline Mean weight, kg & $15.8(15.4-16.2)$ & $14.7(14.3-15.2)$ & $15.4(15.1-15.7)$ & $<0.0001$ \\
\hline Mean duration of illness, days & $2.8(2.6-2.9)$ & $3.0(2.8-3.2)$ & $2.8(2.7-2.9)$ & 0.02 \\
\hline Mean temperature, ${ }^{\circ} \mathrm{C}$ & $38.2(38.1-38.2)$ & $38.1(38-38.2)$ & $38.2(38.1-38.2$ & 0.65 \\
\hline Mean haematocrit level, $\%$ & $31(30.6-31.4)$ & $30.4(30-30.9)$ & $30.8(30.5-31.1)$ & 0.06 \\
\hline Haematocrit level <30\%, $n$ & $237(34 \%)$ & $186(38 \%)$ & $423(36 \%)$ & 0.23 \\
\hline \multicolumn{5}{|l|}{ Parasitaemia, $/ \mu \mathrm{L}$} \\
\hline Geometric mean & 31,694 & 25,080 & 28,756 & 0.01 \\
\hline Range & $1,018-1,096,636$ & $1,030-2,124,000$ & $1,018-2,124,000$ & \\
\hline Parasitaemia $>250,000 / \mu \mathrm{L}, n$ & $37(5 \%)$ & $18(4 \%)$ & $55(5 \%)$ & 0.22 \\
\hline
\end{tabular}

Values express mean (95\% confidence interval), unless otherwise indicated. AA, artesunate-amodiaquine; AL, artemether-lumefantrine.

flank, i.e., 164/332 (49\%), than in those from sites located on the western flank, i.e., $129 / 324(39 \%)(p=0.01)$. On day 28 , the frequencies had decreased significantly to $10 / 109$ (9.2\%), 19/106 (17.9\%), 8/109 (7.3\%), 8/112 (7.1\%), 2/103 (1.9\%), and 4/90 (4.4\%) in Damboa, Agbani, Barkin Ladi, Makarfi, and the Sabo quarters of Ibadan and Ijede, respectively (test for trend: $p<0.0001$ for each of the sites).

In the later study, overall, at presentation, 130/535 $(24.3 \%)$, i.e., $50 / 149(34 \%)$ and $80 / 386(21 \%)$ children aged $<5$ and $5-10$ years, respectively, were anaemic. The difference between the 2 proportions was significant $(p=$ 0.003 ). After the initiation of treatment, the differences in the frequencies of anaemia in children aged $<5$ and 5-10 years were significant on the following follow-up days: day $1(69 / 133$ [51.9\%] vs. $106 / 340$ [31.2\%]; $p<0.0001)$, day $14(30 / 108[40.1 \%]$ vs. $42 / 301[13.9 \%] ; p=0.002)$, day $21(15 / 103$ [14.6\%] vs. $18 / 273$ [4.1\%]; $p=0.03)$, and day $42(9 / 103[8.7 \%]$ vs. $7 / 275[2.5 \%] ; p=0.02)$, respectively. On day 42 , the overall frequency of anaemia was $16 / 378$ (4.2\%). The corresponding frequencies of anaemia in children $<5$ and $5-10$ years of age were $9 / 103(8.7 \%)$ and $7 / 275$ (2.5\%), respectively (test for trend: $p<0.0001$ for overall and the 2 age groups).

The frequencies of anaemia before and after treatment initiation were similar in the 2 treatment groups in both the initial and the later study (data not shown). In the later study, the frequency of anaemia at presentation did not decrease over a 6-year period, i.e., 58/213 [27\%], $31 / 156$ [20\%], 11/51 [22\%], 12/54 [22\%], 5/23 [22\%], and $13 / 38$ [34\%] in 2009, 2010, 2011, 2012, 2013, and 2014, respectively $\left(\chi^{2}\right.$ for trend $=0.05 ; p=0.82$ ).
The data on haematocrit level per visit day showed that, when taken as a group, the overall temporal change in haematocrit was a decline from normal haematocrit to an early monophasic fall to an anaemia level, followed by a rise to a normal level by day 7 (Table 2), and remaining normal till day 42 (Temporal Pattern 2 [11]). This is similar to the data on non-hyparasitaemic children shown in Figure $2 \mathrm{~h}$. The temporal patterns were similar in the 2 treatment groups (Table 2; figure not shown). Overall, mean haematocrit during follow-up was significantly lower on day 3 than on the other days of follow-up ( $p<$ 0.0001; Table 2). However, the mean haematocrit value on day 3 was similar to that on day $2(p=0.65)$. Post hoc comparison showed that the mean haematocrit value at enrollment was significantly lower than on days 7-42 and significantly higher than on days $1-3$. Table 2 also shows the details of haematocrit values according to drug treatment during the follow-up period. The mean haematocrit value on day 42 was significantly higher in the AA-treated children than in the AL-treated children.

Forty-eight hyperparasitaemic (geometric mean parasitaemia/ $\mu \mathrm{L} 401,090$ [range 252,200-2,124,000]) and 48 non-hyperparasitaemic (geometric mean parasitaemia/ $\mu \mathrm{L} 57,642$ [range 1,636-241,629]) children were matched for similar clinical characteristics and treatment. Following treatment, the fever and parasitaemias cleared within 2 days in both groups. There was no fall in haematocrit in 10 hyperparasitaemic and in 11 non-hyperparasitaemic children. The FIH/1,000 asexual parasites cpb was significantly lower in hyperparasitaemic than in non-hyperparasitaemic children (median 0.008 [range 0.0004-0.028, 
Table 2. Haematocrit values as percentages per visit day in malarious children treated with AA or AL

\begin{tabular}{|c|c|c|c|c|c|c|c|c|c|c|}
\hline \multirow[t]{2}{*}{ Drug } & \multicolumn{10}{|c|}{ Follow-up day } \\
\hline & 0 & 1 & 2 & 3 & 7 & 14 & 21 & 28 & 35 & 42 \\
\hline $\mathrm{AA}$ & $\begin{array}{l}31 \pm 5 \\
{[14-47]} \\
30.6-31.4 \\
(n=696)\end{array}$ & $\begin{array}{l}30.4 \pm 4.5 \\
{[17-42]} \\
30-30.8 \\
(n=488)\end{array}$ & $\begin{array}{l}29.7 \pm 4.4 \\
{[16-44]} \\
29.3-30.1 \\
(n=479)\end{array}$ & $\begin{array}{l}29.6 \pm 4.5 \\
{[16-43]} \\
29.3-30 \\
(n=538)\end{array}$ & $\begin{array}{l}30.5 \pm 4.4 \\
{[14-54]} \\
30.1-30.8 \\
(n=627)\end{array}$ & $\begin{array}{l}32.3 \pm 4.1 \\
{[19-50]} \\
32-32.7 \\
(n=600)\end{array}$ & $\begin{array}{l}33.4 \pm 3.5 \\
{[20-45]} \\
33.1-33.7 \\
(n=502)\end{array}$ & $\begin{array}{l}34.2 \pm 3.7 \\
{[20-53]} \\
33.9-34.5 \\
(n=575)\end{array}$ & $\begin{array}{l}34 \pm 3 \\
{[26-44]} \\
33.6-34.3 \\
(n=292)\end{array}$ & $\begin{array}{l}34.3 \pm 3.2 \\
{[20-45]} \\
34-34.7 \\
(n=317)\end{array}$ \\
\hline $\mathrm{AL}$ & $\begin{array}{l}30.4 \pm 4.9 \\
{[16-44]} \\
30-30.9 \\
(n=495)\end{array}$ & $\begin{array}{l}30.3 \pm 4.9 \\
{[12-45]} \\
29.7-30.8 \\
(n=309)\end{array}$ & $\begin{array}{l}29.8 \pm 4.8 \\
{[15-40]} \\
29.2-30.3 \\
(n=308)\end{array}$ & $\begin{array}{l}29.6 \pm 4.6 \\
{[13-41]} \\
29.1-30.1 \\
(n=359)\end{array}$ & $\begin{array}{l}30.4 \pm 4.4 \\
{[16-45]} \\
30-30.8 \\
(n=437)\end{array}$ & $\begin{array}{l}32.1 \pm 4.5 \\
{[16-49]} \\
31.7-32.5 \\
(n=422)\end{array}$ & $\begin{array}{l}33.4 \pm 3.9 \\
{[14-45]} \\
33-33.8 \\
(n=348)\end{array}$ & $\begin{array}{l}33.8 \pm 3.9 \\
{[22-50]} \\
33.4-34.2 \\
(n=425)\end{array}$ & $\begin{array}{l}33.7 \pm 3.7 \\
{[20-44]} \\
33.1-34.3 \\
(n=162)\end{array}$ & $\begin{array}{l}33.7 \pm 3.3 \\
{[22-40]} \\
33.1-34.2 \\
(n=165)\end{array}$ \\
\hline ALL & $\begin{array}{l}30.8 \pm 5 \\
{[14-47]} \\
30.5-31.1 \\
(n=1,191)\end{array}$ & $\begin{array}{l}30.3 \pm 4.6 \\
{[12-45]} \\
30-30.7 \\
(n=797)\end{array}$ & $\begin{array}{l}29.7 \pm 4.5 \\
{[15-44]} \\
29.4-30.1 \\
(n=787)\end{array}$ & $\begin{array}{l}29.6 \pm 4.5 \\
{[13-43]} \\
29.3-29.9 \\
(n=897)\end{array}$ & $\begin{array}{l}30.4 \pm 4.4 \\
{[14-54]} \\
30.2-30.7 \\
(n=1,064)\end{array}$ & $\begin{array}{l}32.2 \pm 4.3 \\
{[16-50]} \\
32-32.5 \\
(n=1,022)\end{array}$ & $\begin{array}{l}33.4 \pm 3.7 \\
{[14-45]} \\
33.1-33.6 \\
(n=850)\end{array}$ & $\begin{array}{l}34 \pm 3.8 \\
{[14-53]} \\
33.8-34.3 \\
(n=1,000)\end{array}$ & $\begin{array}{l}33.9 \pm 3.3 \\
{[20-44]} \\
33.6-34.2 \\
(n=454)\end{array}$ & $\begin{array}{l}34.1 \pm 3.3 \\
{[20-45]} \\
33.8-34.4 \\
(n=482)\end{array}$ \\
\hline$p$ value $^{1}$ & 0.06 & 0.72 & 0.89 & 0.85 & 0.78 & 0.39 & 0.95 & 0.14 & 0.43 & 0.02 \\
\hline
\end{tabular}

Values express mean \pm standard deviation, [range], and 95\% confidence interval. AA, artesunate-amodiaquine; AL, artemetherlumefantrine; ALL, all children.

${ }^{1}$ For comparison of means of AA and AL treatment groups.

$n=38$ ] vs. median 0.028 [range $0.0016-1.22, n=37$ ], $p<$ $0.0001)$. Similarly, on days $0-2$, the average haematocrit: total parasitaemia cleared was significantly lower in hyperparasitaemic than in non-hyperparasitaemic children (median 0.00009 [range 0.00001-0.00014] vs. median 0.00035 [range 0.00012-0.021], $p<0.0001$ ). FIH/1,000 asexual parasites $\mathrm{cpb}$ and average haematocrit:total parasitaemia cleared (on days 0-2) were similar in children treated with $\mathrm{AA}$ and in those treated with $\mathrm{AL}$ (data not shown).

The overall temporal patterns of change in haematocrit levels were similar in hyperparasitaemic and non-hyperparasitaemic children, (Fig. 2h) and Pattern 2 (Fig. 2b), and similar to the overall pattern of haematocrit levels per visit day in all of the malarious children. The frequencies of the patterns were similar with both treatments. A late monophasic fall, e.g., Pattern 3, (Fig. 2c, $n=4$ ) and Pattern 7 (Fig. 2g, $\mathrm{n}=1$ ) as well as early and late monophasic falls, e.g., Pattern 4 (Fig. 2d, $n=1$ ), occurred in 6 children with or without hyperparasitaemia. All 6 children with late monophasic falls in haematocrit to $<30 \%$ (i.e., Patterns 3,4 , and 7 above) had parasitaemias $>180,000 / \mu \mathrm{L}$ at presentation. Four of 6 children cleared their parasitaemias within 1 day of starting treatment, 5 of 6 children were treated with AA, with a total dose of artesunate $\geq 10$ $\mathrm{mg} / \mathrm{kg}$, and all 6 were asymptomatic when the late fall in haematocrit occurred. In addition, FIH/1,000 asexual

Anaemia following Artemisinin-Based Combination Treatments parasites $\mathrm{cpb}$ and the average haematocrit:total parasitaemia cleared (on days $0-2$ ) were very low in these children.

Assessment data on haematocrit levels before and after the initiation of treatment in matched hyperparasitaemic and non-hyperparsitaemic children was similar (data not shown). On day 28, overall, the haematocrit level was $33.4 \%$ (range $24-45 \%$ ), and it was similar in hyperparasitaemic and non-hyperparasitaemic children $(32.7 \%$ [range $24-38$ ] vs. $34.1 \%$ [range $24-45$ ], $p=0.07$ ). On day 35 , overall, the haematocrit level was $33.8 \%$ (range $24-$ $42 \%$ ), and it was significantly lower in hyperparasitaemic than in non-hyperparasitaemic children $(32.7 \%$ [range $24-39$ ] vs. $34.6 \%$ [range 29-42], $p=0.03$ ). On day 42 , haematocrit levels were similar in both groups of children (Fig. 2h). In children who were hyperparasitaemic and anaemic at presentation, the median FIH/1,000 asexual parasites $\mathrm{cpb}$ was significantly lower than in non-hyperparasitaemic and anaemic children at presentation (median 0.004 [range $0.0002-0.023, n=10$ ] vs. median 0.031 [range $0.019-0.21, n=4$ ], $p=0.030$ ). Figure $2 \mathrm{f}$ shows an absence of precipitous falls in haematocrit during the first few days following treatment in children with hyperparasitaemia and anaemia. Similarly, in children who were hyperparasitaemic and anaemic at enrollment, the average haematocrit:total parasitaemia cleared (on days 0-2) was significantly lower than in non-hyperparasitaemic and anaemic children (median 0.00007 [range 0.000013-

Chemotherapy 2017;62:231-238 DOI: $10.1159 / 000449366$ 


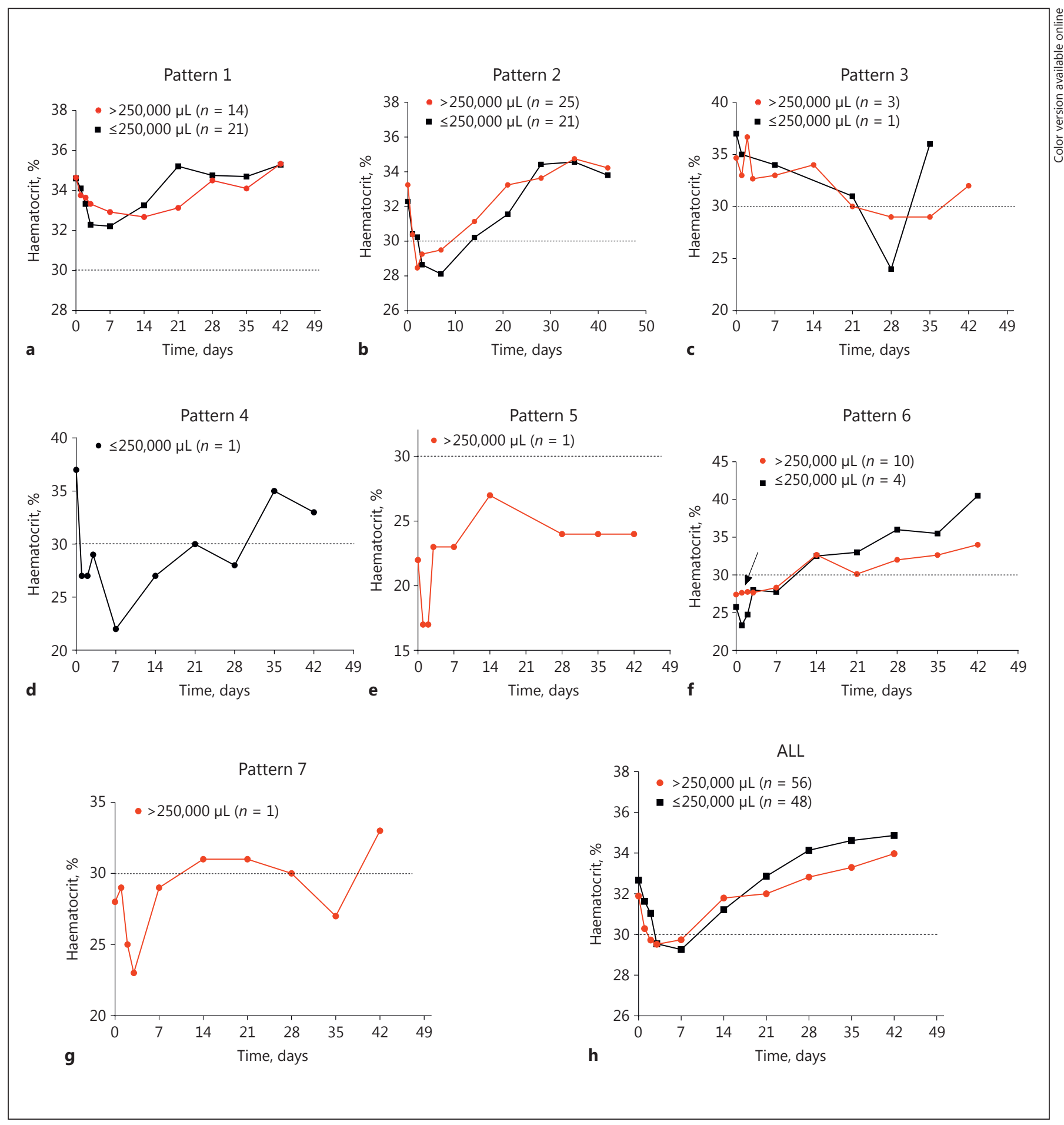

Fig. 2. a-h Temporal patterns of haematocrit in hyperparasitaemic (red line) and in age, gender, same-treatment, and same-day presentation-matched non-hyperparasitaemic children (black line) treated with artesunate-amodiaquine (AA) or artemether-lume- fantrine (AL). Arrow indicates absence of dip in haematocrit level in children with hyperparasitaemia and anaemia at presentation (description of patterns [11]). ALL, all children (i.e., with AA or $\mathrm{AL})$. 
$0.00011, n=10$ ] vs. median 0.00021 [range 0.00014 $0.00581, n=4], p=0.002$ ).

Twenty-one of 330 children (222 and 108 children treated with $\mathrm{AA}$ and $\mathrm{AL}$, respectively), met the criteria for the diagnosis of late-appearing anaemia; 13 and 8 of these were treated with AA and AL, respectively. In the 21 children with late-appearing anaemia, the mean age was 5.4 (range 1-10) years, and geometric mean parasitaemia was $63,611 / \mu \mathrm{L}$ (range 2,880-618,950); in 9 children, parasitaemia was $>100,000 / \mu \mathrm{L}$, and parasitaemia cleared within 1 day of starting treatment in 17 children. The mean time to late-appearing anaemia was 27 days (range 21-42 days). Nadir haematocrit was 26.5\% (range 20-29\%). All but 1 of the 21 children were asymptomatic when late-appearing anaemia occurred. The only exception was an 8 -year-old female child who had fever on day 28 , when late-appearing anaemia occurred. All 6 children with a late monophasic fall to an anaemia level met the clinical and parasitological criteria for the diagnosis of late-appearing anaemia.

Of the 48 pairs of matched hyperparasitaemic and non-hyperparasitaemic children (i.e., a total of 96 children), 6 children ( 5 hyperparasitaemic and 1 non-hyperparsitaemic) had late monophasic, or both early and late monophasic falls in haematocrit to an anaemia level (see above: temporal patterns of haematocrit in these 6 children). In these 6 children, the mean age was 4.7 (range 3-6) years, and geometric mean parasitaemia was 304,835 / $\mu \mathrm{L}$ (range 181,500-618,950); in 4 children, parasitaemia cleared within 1 day of starting treatment (mean parasite clearance time was 1.33 days). Mean haematocrit level at enrollment was $34.3 \%$ (range $28-40 \%$ ). Mean time to late-appearing anaemia was 30 days (range 21-35 days). Mean nadir haematocrit was $25.5 \%$ (range 20-29\%). None of the children was asymptomatic when late-appearing anaemia occurred. Mean FIH/1,000 parasites cpb was 0.0186 (range $0.0074-0.0551$ ). Mean ratio of average haematocrit:total parasitaemia cleared (on days $0-2$ ) was 0.00019 (range 0.00006-0.0006). The temporal patterns of change in haematocrit in the 6 children were Patterns 3,4 , or 7 (see above: temporal patterns of haematocrit). The overall plot of these patterns was the same as that of hyperparasitaemic children shown in Figure $2 \mathrm{~h}$.

\section{Discussion}

In this study, both AA and AL significantly reduced the frequency of anaemia in younger and older malarious children following treatment. However, it is unclear why the frequency of anaemia at presentation was significant-

Anaemia following Artemisinin-Based

Combination Treatments ly higher at the sites located on the eastern flank of the study. Differences in the background causes of anaemia (e.g., helminth infections, immunoglobinopathies, glucose-6-phosphate dehydrogenase deficiency, and nutritional factors) as well as other unidentified factors may be responsible for the significant difference in the frequency of anaemia between the eastern and the western flanks of the study sites.

Estimates of the indicators of haematocrit conservation revealed that it was much more pronounced in matched hyperparasitaemic children than in non-hyperparasitaemic children, with further evidence for this being seen in the similar temporal patterns of haematocrit (Fig. 2h) and haematocrit levels in the first week of treatment. These findings confirm the prevention of precipitous falls in haematocrit in hyperparasitaemic children. The absence of a dip in haematocrit in hyperparasitaemic children with anaemia in the first week (Fig. 2f) suggests that AA and AL also prevent precipitous falls or attenuate the falls in haematocrit in these 2 conditions in malarious children.

Despite the similar haematocrit profiles in the first week of treatment in hyperparasitaemic and non-hyperparasitaemic children, mean haematocrit levels were significantly lower in hyperparasitaemic than in non-hyperparasitaemic children 5 weeks after treatment began (Fig. 2h). This would suggest that the initial haematocrit "conserved" early in treatment in children with hyperparasitaemia was later lost. However, this does not prove that it is the once-infected erythrocytes that were later destroyed 5 weeks after treatment began. It is possible that other factors may have contributed to the later decline in haematocrit, e.g., the destruction of normal red blood cells.

Falls in haematocrit to a level of anaemia are characteristics of late monophasic falls only; these occurred in $6 \%$ of the cohort of 104 hyperparasitaemic and non-hyperparasitaemic children evaluated. The clinical and parasitological features of these children were somewhat similar to those of 15 other children who met the clinical and parasitological criteria for the diagnosis of late-appearing anaemia. Indeed, all children with late monophasic falls in haematocrit to an anaemia level met the criteria for the diagnosis of late-appearing anaemia, suggesting that these terms can be used interchangeably. In these children, the development of late-appearing anaemia was accompanied by few or no symptoms. It is important to note that the absence of symptoms when late-appearing anaemia occurs and the frequent use of ACTs in an already anaemic child may lead to the development of chronic anaemia [12].

Chemotherapy 2017;62:231-238

DOI: $10.1159 / 000449366$ 
Late-appearing anaemia superficially resembles delayed haemolytic anaemia following the treatment of severe malaria with intravenous artesunate in immunologically naïve patients $[3,4]$. However, late-appearing anaemia differs from post-artesunate delayed haemolysis $(\mathrm{PADH})$ syndrome $[3,15]$ in the relative absence of symptoms and overt clinical and laboratory features of haemolysis. In this context, the limitations of our study are: not quantifying the once-infected red blood cells, not evaluating the laboratory parameters for the confirmation of a haemolytic or non-haemolytic nature of the late-appearing anaemia, and not evaluating the contribution of the background causes of anaemia in children from this endemic area to the various patterns of temporal change in haematocrit to $<30 \%$ following the AA and AL treatments.

In conclusion, AA and AL significantly reduce the frequency of anaemia, conserve haematocrit at high parasitaemias with or without accompanying anaemia, and may cause a late monophasic fall in haematocrit to $<30 \%$ or a late-appearing anaemia in about $6 \%$ of malarious children 3-5 weeks after the start of treatment.

\section{Acknowledgements}

We thank all participating children and their parents/guardians. The therapeutic efficacy studies from which the data was derived received financial support from Swiss Pharmaceuticals Nigeria PLC, the World Bank Malaria Booster Project, and the Global Fund for Malaria. Logistic support for the study was partly provided by Society for Family Health and Support for National Malaria Programs (SunMap) in Nigeria.

\section{Disclosure Statement}

The authors declare that they have no competing interests.

\section{Author Contributions}

A.S. conceived and designed the study, gathered and analysed data and wrote the manuscript. K.A. gathered and analysed data and wrote the manuscript. C.A. analysed data: G.N., A.A., S.O., H.O., I.W., M.M., W.O. P.A., O.A., B.F., T.A., C.H., G.G., and O.F. gathered and generated data. All authors read and approved the final version of the manuscript.

\section{References}

1 Bosman A, Mendis KN: A major transition in malaria treatment: the adoption and deployment of artemisinin-based combination therapies. Am J Trop Med Hyg 2007;7(suppl 6):193-197.

2 World Health Organization: Guidelines for the Treatment of Malaria, ed 2. Geneva, 2010.

3 Jauréguiberry S, Ndour PA, Roussel C, Ader F, Safeukui I, Nguyen M, Biligui S, Ciceron L, Mouri O, Kendjo E, Vray M, Angoulvant A, Mayaux J, Haldar K, Mazier D, Danis M, Caumes E, Thellier M, Buffet P: Postartesunate delayed hemolysis is a predictable event related to the lifesaving effect of artemisinins. Blood 2014;124:167-175.

4 Medicine for Malaria Venture: Experts group meeting on delayed haemolytic anaemia following treatment with injectable artesunate. Vienna, 2013. http:/www.mmv.org/newsroom/events/expert-group-meeting-safetyprofile-injectable-artesunate.

5 Gbotosho GO, Okuboyejo TM, Happi CT, Sowunmi A: Fall in hematocrit per 1,000 parasites cleared from peripheral blood: a simple method for estimating drug-related fall in hematocrit after treatment of malaria infections. Am J Ther 2014;21:193-197.
6 Gbotosho GO, Sowunmi A, Okuboyejo TM, Happi CT: Oral artesunate-amodiaquine and artemether-lumefantrine in the treatment of uncomplicated hyperparasitaemic Plasmodium falciparum malaria in children. J Trop Pediatr 2012;58:151-153.

7 Luxemburger C, Nosten F, Raimond SD, Chongsuphajaisiddhi T, White NJ: Oral artesunate in the treatment of uncomplicated hyperparasitaemic P. falciparum malaria. Am J Trop Med Hyg 1995;53:522-525.

8 Sowunmi A, Okuboyejo TM, Gbotosho GO, Happi CT: Risk factors for gametocyte carriage in uncomplicated $P$. falciparum malaria in children before and after artemisinin-based combination treatments. Chemotherapy 2011;57:497-504.

9 Oguche S, Okafor HU, Watila I, Meremikwu M, Agomo P, Ogala W, Agomo C, Ntadom G, Banjo O, Okuboyejo T, Ogunrinde G, Odey F, Aina O, Sofola T, Sowunmi A: Efficacy of artemisinin-based combination treatments of uncomplicated $P$. falciparum malaria in under-five-year-old Nigerian children. Am J Trop Med Hyg 2014;91:925-935.
10 World Health Organization: Assessment and Monitoring of Antimalarial Drug Efficacy for the Treatment of Uncomplicated $P$. falciparum Malaria. Geneva, 2003.

11 Sowunmi A, Akano K, Ayede AI, Ntadom G, Fatunmbi B, Aderoyeje T, Adewoye EO: Temporal changes in haematocrit following artemisinin-based combination treatments of uncomplicated $P$. falciparum malaria in children. BMC Inf Dis 2015;15:454.

12 Sowunmi A, Akano K, Ayede AI, Ntadom G, Aderoyeje T, Adewoye EO, Fatunmbi B: Clinical illness and outcomes in Nigerian children with late-appearing anaemia after artemisinin-based combination treatments of uncomplicated $P$. falciparum malaria. BMC Inf Dis 2016;16:240.

13 Centers for Disease Control and Prevention: Epi Info ${ }^{\mathrm{TM}}$ version 7.0. A Word-Processing Database and Statistics Programme for Public Health on IBM-Compatible Microcomputers. Atlanta, 2013.

14 SPSS Inc.: SPSS for Windows release 20.0 (standard version). Chicago, 2011.

15 Arguin PM: Case definition: postartemisinin delayed haemolysis. Blood 2014;124:157-158. 